University of Wollongong

Research Online

Faculty of Engineering and Information

Faculty of Engineering and Information

Sciences - Papers: Part A

Sciences

2016

High-throughput, sheathless, magnetophoretic separation of magnetic and non-magnetic particles with a groove-based channel

\author{
Sheng Yan \\ University of Wollongong, sy034@uowmail.edu.au \\ Jie Zhang \\ University of Wollongong, jz248@uowmail.edu.au \\ Dan Yuan \\ University of Wollongong, dy983@uowmail.edu.au \\ Qianbin Zhao \\ University of Wollongong, qz260@uowmail.edu.au \\ $\mathrm{J} \mathrm{Ma}$ \\ University of Science and Technology of China
}

See next page for additional authors

Follow this and additional works at: https://ro.uow.edu.au/eispapers

Part of the Engineering Commons, and the Science and Technology Studies Commons

Research Online is the open access institutional repository for the University of Wollongong. For further information contact the UOW Library: research-pubs@uow.edu.au 


\title{
High-throughput, sheathless, magnetophoretic separation of magnetic and non- magnetic particles with a groove-based channel
}

\author{
Abstract \\ The separation of target objects conjugated with magnetic particles is a significant application in \\ biomedicine and clinical diagnosis. Conventional magnetophoresis-based devices use a sheath flow to \\ pre-focus the particles into a single stream and typically operate at a low flow rate. We demonstrate in \\ this work a high-throughput, sheathless, magnetophoretic separation of magnetic and non-magnetic \\ beads in a groove-based channel, and also report on an interesting phenomenon where the same \\ magnetic beads in the same microchannel, but with different setups, has a different particle tracing; a \\ binary mixture of magnetic and non-magnetic beads in a diluted ferrofluid, is then fed into the channel. \\ These magnetic beads are focused near the centreline of the channel by exploiting positive \\ magnetophoresis and microvortices generated by grooves, whereas the non-magnetic beads are focused \\ along the sidewalls of the channel by negative magnetophoresis and hydrophoresis. These magnetic and \\ non-magnetic beads are separated in a wide range of flow rates (up to $80 \mu \mathrm{l} \mathrm{min}^{-1}$ ). \\ Disciplines \\ Engineering | Science and Technology Studies

\section{Publication Details} \\ Yan, S., Zhang, J., Yuan, D., Zhao, Q., Ma, J. \& Li, W. H. (2016). High-throughput, sheathless, \\ magnetophoretic separation of magnetic and non-magnetic particles with a groove-based channel. \\ Applied Physics Letters, 109 (21), 214101-1-214101-5.

\section{Authors} \\ Sheng Yan, Jie Zhang, Dan Yuan, Qianbin Zhao, J Ma, and Weihua Li
}




\title{
High-throughput, sheathless, magnetophoretic separation of magnetic and non-magnetic particles with a groove-based channel
}

\author{
S. Yan, ${ }^{1}$ J. Zhang, ${ }^{1,2}$ D. Yuan, ${ }^{1}$ Q. Zhao, ${ }^{1}$ J. Ma,${ }^{3}$ and W. H. Li ${ }^{1, a)}$ \\ ${ }^{1}$ School of Mechanical, Materials and Mechatronic Engineering, University of Wollongong, Wollongong, \\ NSW 2522, Australia \\ ${ }^{2}$ School of Mechanical Engineering, Nanjing University of Science and Technology, Nanjing 210094, China \\ ${ }^{3}$ Department of Precision Machinery and Instrumentation, University of Science and Technology of China, \\ Hefei 230031, China
}

(Received 27 August 2016; accepted 14 November 2016; published online 23 November 2016)

\begin{abstract}
The separation of target objects conjugated with magnetic particles is a significant application in biomedicine and clinical diagnosis. Conventional magnetophoresis-based devices use a sheath flow to pre-focus the particles into a single stream and typically operate at a low flow rate. We demonstrate in this work a high-throughput, sheathless, magnetophoretic separation of magnetic and nonmagnetic beads in a groove-based channel, and also report on an interesting phenomenon where the same magnetic beads in the same microchannel, but with different setups, has a different particle tracing; a binary mixture of magnetic and non-magnetic beads in a diluted ferrofluid, is then fed into the channel. These magnetic beads are focused near the centreline of the channel by exploiting positive magnetophoresis and microvortices generated by grooves, whereas the non-magnetic beads are focused along the sidewalls of the channel by negative magnetophoresis and hydrophoresis. These magnetic and non-magnetic beads are separated in a wide range of flow rates (up to $\left.80 \mu \mathrm{l} \mathrm{min}^{-1}\right)$. Published by AIP Publishing. [http://dx.doi.org/10.1063/1.4968835]
\end{abstract}

Bead-based immunoassays in microfluidics enable biological samples ${ }^{1,2}$ to be detected and separated more accurately. Conjugation with beads can amplify the volume of target objects (e.g., cells, DNA, protein) via specific antibody-antigen reactions and also change their magnetic and dielectric properties, and the density. ${ }^{3}$ By tagging target cells with immuno-labelled beads to enhance their size, the target cells can be separated from non-target cells of a comparable size. ${ }^{4,5}$ Elastomeric beads are popular in acoustophoretic devices because they can make the tagged target cells less dense. ${ }^{6}$ In addition, polystyrene particles are used to bind with target objects so that the bound complexes show a different dielectrophoretic behaviour in an alternate field and are thus separated from non-labelled objects. ${ }^{7}$ However, cells of interest that are labelled with immunomagnetic beads to be handled in a magnetic field are called "positive magnetophoresis." Due to their non-contact nature, the viability of cells can be secured by avoiding potential hazards, and since magnetic separation is not influenced very much by any changes in the chemical or thermal characteristics of the fluid carrier, separating the target cells that have conjugated with magnetic beads, can be simplified into separating magnetic particles from non-magnetic particles.

Several microfluidic devices that will separate magnetic from non-magnetic particles have been reported. ${ }^{8}$ Magnetophoretic separation normally requires a sheath flow to pre-focus the samples containing paramagnetic beads and diamagnetic beads, and then the paramagnetic beads are deflected from the initial fluid stream in a magnetic field. Zhu et al. ${ }^{9}$ used permanent magnets to separate magnetic and nonmagnetic particles of the same size under a maximum flow rate of $3 \mu \mathrm{lmin}^{-1}$. Xia et al. ${ }^{10}$ integrated micro-comb and

${ }^{a}$ E-mail: weihuali@uow.edu.au micro-needle structures to enhance the magnetic field, after which non-magnetic beads ( $2.0 \mu \mathrm{m}$ in diameter) and magnetic beads ( $1.6 \mu \mathrm{m}$ in diameter) were separated with an efficiency

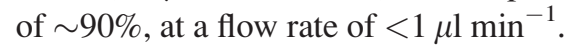

In all these studies, a sheath flow is needed for separation because particle suspension is pushed into one side of the channel and the magnetic beads are deflected laterally from non-magnetic beads under a magnetic field. To improve the displacement of magnetic beads means increasing the exposure time to the magnetic field because it decreases sharply as the distance from the magnetic source increases. ${ }^{11}$ All these factors lead to a low flow rate in magnetophoresisbased chips. Moreover, sheath flow requires an accurate flow control because any unstable pumping means the particles will not be confined perfectly and will be deflected from their original positions. ${ }^{12}$ This is why a high-throughput, sheathless magnetophoresis device is needed to address these issues. If magnetic beads migrated vertically, the width of the channel would have almost no effect on their deflection, so increasing the width of the channel will dramatically improve their throughput. Moreover, this type of magnetophoresis can be joined with other hydrodynamic effects to achieve a sheathless particle separation.

Several sheathless techniques for separating particles in microfluidics have been reported in the literature review, ${ }^{13}$ of which the groove-based channel can separate particles with a different position in the height direction. A groovebased channel for mixing was first proposed by Stroock et al. ${ }^{14}$ using a vortex; this was then developed for trapping by Gadish and Voldman, ${ }^{15}$ using a combination of microvortex and dielectrophoretic phenomenon. Later, Hsu et al. ${ }^{16}$ used the microvortex generated by grooves to separate a binary mixture of beads with differing densities. 
The aim here is to develop a microfluidic device that couples magnetophoresis and microvortex for a highthroughput, sheathless separation of magnetic beads from non-magnetic beads. Magnetic and non-magnetic beads are suspended in a diluted ferrofluid and located at different equilibrium positions by balancing magnetophoretic and drag forces without sheath flow (Figs. 1(a) and 1(b)). Throughput can be improved significantly by widening the channel, even though it is not related particle deflection in a vertical direction. This work combines magnetophoresis and microvortex in order to have a stable separation of target objects from non-target ones.

Microfluidics devices are fabricated by two-step photolithography and soft lithography. Fig. 1(c) shows a top view of the $600 \mu \mathrm{m}$ wide by $2 \mathrm{~cm}$ long channel with 60 grooves, each of which has a small $300 \mu \mathrm{m}$ curvature and a large curvature of $350 \mu \mathrm{m}$. Each layer of the SU-8 mould is $40 \mu \mathrm{m}$ high. Details regarding the fabrication, materials, and experimental setup of this device are available in the supplementary material.

The particles in this device experience two primary forces: a drag force induced by secondary flow, and a magnetophoretic force. The drag force in the $z$-axis is expressed as $F_{d z}=3 \pi d \eta v_{z}$, where $d$ is the diameter of the particle, $\eta$ is the viscosity of the medium, and $v_{z}$ is the $z$-velocity of the flow. ${ }^{17}$ The magnetic force exerting on a magnetised body in a suspending fluid under a non-uniform magnetic field is expressed as ${ }^{9}$

$$
\mathrm{F}_{\mathrm{mag}}=\mu_{0} V_{p}\left[\left(\mathrm{M}_{\mathrm{p}}-\mathrm{M}_{\mathrm{f}}\right) \cdot \nabla\right] \mathrm{H}
$$

where $\mu_{0}$ is the permeability of free space $\left(4 \pi \times 10^{-7} \mathrm{H}\right.$ $\left.\mathrm{m}^{-1}\right), V_{p}$ is the volume of the particle, $\mathbf{M}_{\mathbf{p}}$ is the magnetization of the magnetized particle, $\mathbf{M}_{\mathbf{f}}$ is the magnetization of the fluid, and $\mathbf{H}$ is the magnetic field at the center of the particle. Eq. (1) states that particles of different size, $V_{p}$, and/or magnetizations, $\mathbf{M}_{\mathbf{p}}=\chi_{p} \mathbf{H}$ with $\chi_{p}$ being the dimensionless magnetic susceptibility of the particle, experience different
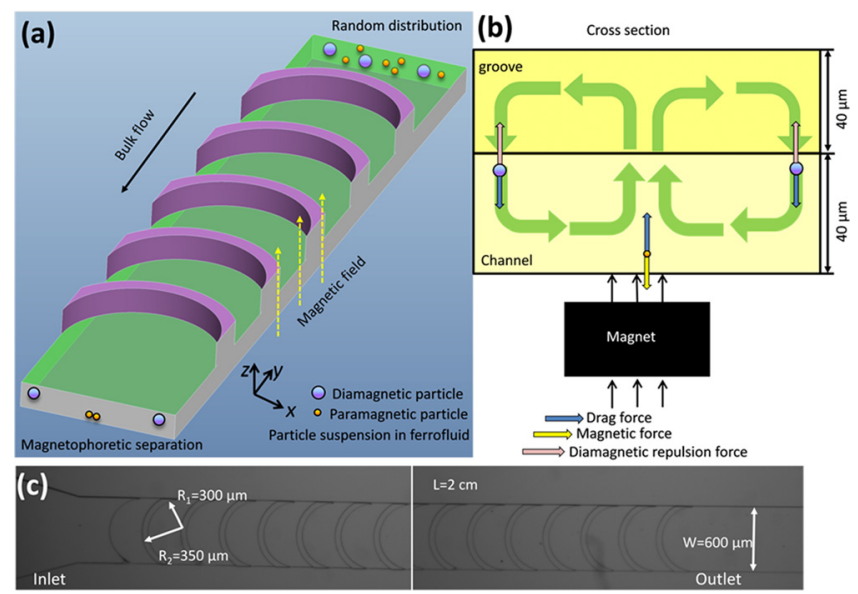

FIG. 1. A microfluidic device for separating magnetic and non-magnetic particles. (a) Schematic showing the structure of this device and the spatial distributions of particles. The magnetic particles migrate to the centreline of the channel, while non-magnetic particles are focused onto the sidewalls. (b) The cross-sectional force diagram and equilibrium locations of particles in a microchannel patterned with grooves on its ceiling. (c) Optical micrograph images of the groove-based channel. magnetophoretic forces in the same suspending fluid $\left(\mathbf{M}_{\mathbf{f}}=\chi_{f} \mathbf{H}\right.$ with $\chi_{f}$ being the magnetic susceptibility of the fluid). Eq. (1) can be expressed in another form ${ }^{18}$

$$
\mathrm{F}_{\mathrm{mag}}=\mu_{0}\left(\chi_{p}-\chi_{m}\right) \mathrm{V}_{\mathrm{p}}(\mathrm{H} \cdot \nabla) \mathrm{H}
$$

For a magnetic particle $\left(\chi_{p 1}>0\right)$ dispersed in an aqueous diamagnetic medium $\left(\chi_{m}<0\right)$, a positive magnetophoresis is generated and the particles migrate to the region of a strong magnetic field, but if the particles are diamagnetic $\left(\chi_{p 2}<0\right)$ and the medium is paramagnetic $\left(\chi_{m}>0\right)$ then the difference between the values becomes negative and the particles are repelled from the magnetic field towards an area of field minima. In our study, the magnetic and non-magnetic beads were prepared in $\times 0.05$ EMG 408 ferrofluid in which $\chi_{p 1}>\chi_{m}>\chi_{p 2}$; this is where, the magnetic beads experience a positive magnetophoresis, and the diamagnetic beads exert magnetic repulsion forces.

The equilibrium of magnetic beads (indicated as an orange sphere in Fig. 1(b)) is located at the centreline of the channel where two adjacent counter-rotating microvortices converge. The magnetic beads are attracted towards the magnet by the balancing magnetic force and vertical drag force. In a lateral direction (along the $x$-axis), the drag forces are balanced but non-magnetic particles have two "trap sites" located near the sidewalls, so the magnetic repulsion forces push particles upwards to the upper level of the channel where particle-groove interactions enable hydrophoretic focusing. ${ }^{19}$

Since arranging a set of two neodymium-iron-boron $(\mathrm{NdFeB})$ magnets under the microchannel will block any view of the inverted microscope, the chip is flipped so that the magnets can be placed on top of the glass side (Fig. 2(a)). The particle suspension flows through the channel via lateral ports, while the fabrication of these lateral fluidic ports was reported in our previous work. ${ }^{19}$ This device is called a "flipped" setup, and consists of magnets, glass, and polydimethylsiloxane (PDMS) with a groove-based channel, from top to bottom (Fig. 2(b)). The magnetic beads evenly distributed in the inlet are gradually focused at the centreline of the channel by a balance of magnetic force and drag force.

To verify that the magnetic beads are focusing in the groove-based channel, with help from the magnetic field, the magnetic beads suspended in distilled (DI) water were injected into the channel. Fig. 2(c) shows the particle trajectories of $6 \mu \mathrm{m}$ magnetic beads captured at a flow rate of $1 \mu \mathrm{l}$ $\min ^{-1}$. Regardless of their original positions, all the beads were focused on the centreline of the channel. The magnetic force then attracted the magnetic beads to the top of the channel, where drag forces gradually forced the beads into a single line moving in a lateral direction.

Fig. 2(d) shows the bright-field microscopy of magnetic beads focused in the groove-based channel under various flow rates. The focusing performance is quantified by introducing the focusing efficiency as: ${ }^{20} \mathrm{E}_{\mathrm{n}}=(w-d / 2-a) /(w-d)$, where $a$ represents the width of the focused particle stream. The focusing efficiency versus flow rate is plotted and presented in Fig. 2(e). Focusing efficiency increased ( $>95 \%$ ) when the flow rate is less than $30 \mu \mathrm{l} \mathrm{min}{ }^{-1}$, but the focusing efficiency decreased from $92.0 \pm 2.9 \%$ to $87.1 \pm 3.6 \%$ when the flow rate increased from 50 to $100 \mu \mathrm{l} \mathrm{min}{ }^{-1}$. As Hsu et al. ${ }^{16}$ 

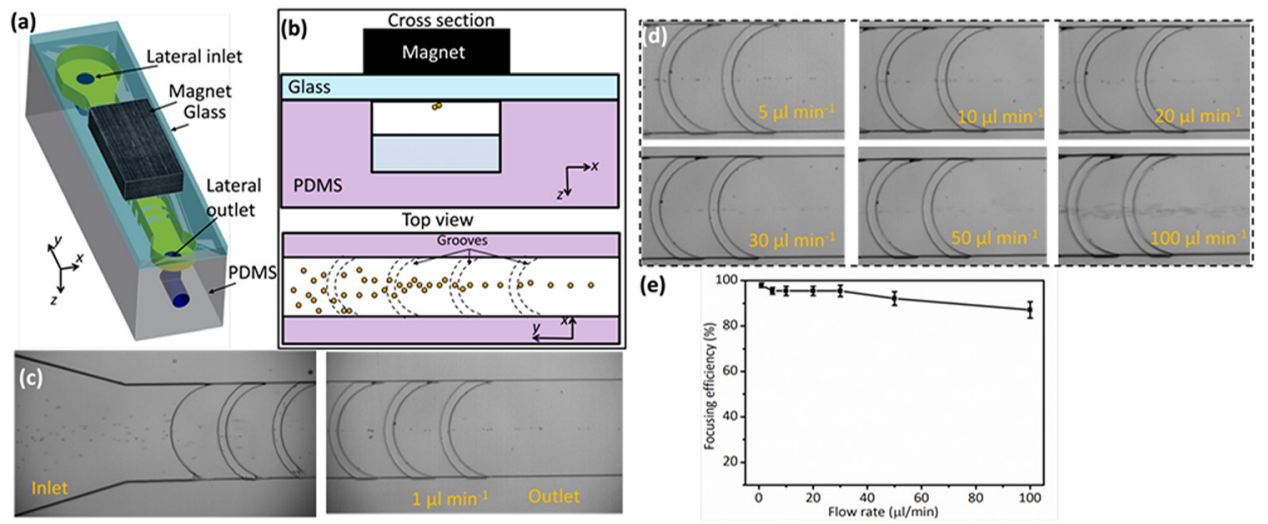

FIG. 2. (a) A three-dimensional animated model with an overview of the "flipped" setup. (b) Cross section (top) and top view (bottom) show that the magnetic beads are gradually focused due to positive magnetophoresis and microvortices. (c) The micrographic images showing the particle trajectories at the inlet (left) and outlet (right) at a flow rate of $1 \mu \mathrm{l} \mathrm{min}^{-1}$. (d) Optical micrographs showing the particle trajectories at the channel outlet at the flow rate of 5, 10, 20, 30, 50 and 100 $\mu \mathrm{lmin}{ }^{-1}$. (e) The measured focusing efficiency of the magnetic beads. The average value was 3 times the measurement and the error bar represents standard deviation.

reported, the drag force in the cross section is linearly proportional to the inlet flow rate for all $\operatorname{Re}<2$, and although the drag force in the cross section decreases at a lower flow rate, the longer retention time in the channel enables the beads to move to the centreline of the channel. However, a larger drag force is induced as the inlet flow rate increases, but the exposure time to the magnetic field is not enough to attract the beads to the top of the channel, and this leads to a slight drop in focusing efficiency.

Despite the focusing efficiency decreasing slightly as the flow rate increases, focusing efficiency remains around $85 \%$ at a maximum flow rate of $100 \mu \mathrm{min}^{-1}$. These higher focusing efficiencies and the wider flow rate range are key parameters for successful separation in the next step. Flow-rate insensitivity is a dispensable characteristic in developing hand-held microfluidic devices where an accurate pumping system is not required. For example, a fluidic sample can be manually introduced into chips via pipettes or syringes. A microfluidic device with an independent flow rate can still achieve good separation or focusing performance, even with a manually operated flow rate that fluctuates and is unstable.

Interestingly, magnetic beads in the "regular" setup have a distinct movement such that a glass slide is placed on the stage of the microscope and the magnets are on top of the PDMS replica (Fig. 3(a)). Similarly, magnetic beads are attracted to the magnetic source (i.e., the upper level of the channel), where they focus onto the sidewalls of the channel due to hydrophoretic self-ordering (Fig. 3(b)). Hydrophoresis has a steric hindrance mechanism which separates or focuses particles or cells under a pressure gradient induced by the grooves, ${ }^{21}$ so those particles with diameters larger than half the height of the channel will be dominated by steric hindrance and form hydrophoretic ordering. We recently found that particle-groove interaction enables particle focusing, ${ }^{22}$ and these intense particle-groove interactions are achieved by levitating the particles towards the grooves using electric ${ }^{23}$ and magnetic fields, ${ }^{19}$ even though the particles do not satisfy the criterion of hydrophoretic ordering. In this "regular" setup, those magnetic beads that are evenly released are gradually focused onto the sidewalls of the channel by a combination of positive magnetophoresis and hydrophoresis.
Fig. 3(c) shows the focusing patterns of $6 \mu \mathrm{m}$ magnetic beads at the outlet in a "regular" setup where the flow rates vary from 1 to $20 \mu \mathrm{min}^{-1}$. Fig. 3(d) shows the focusing efficiency under different working conditions; in the "regular"
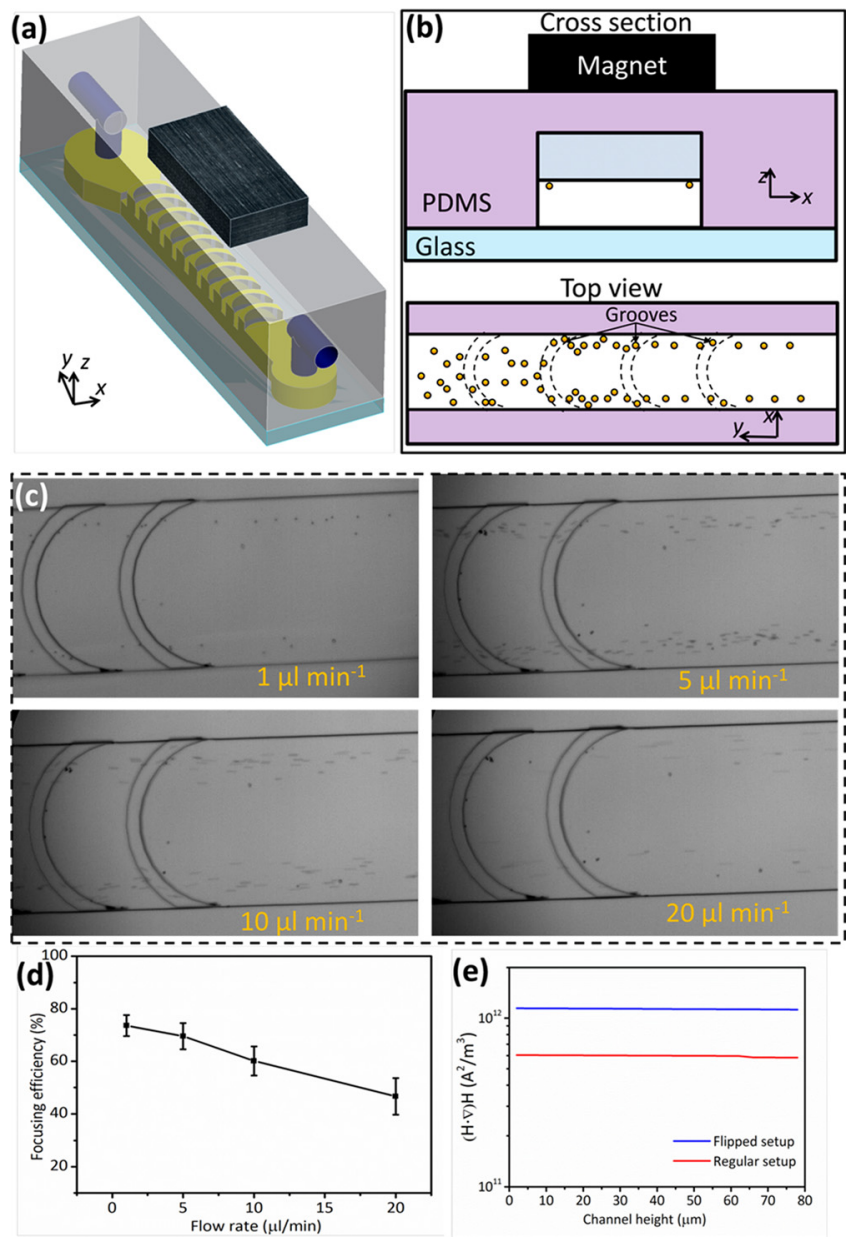

FIG. 3. (a) An overview of the "regular" setup. (b) The cross section (top) and top view (bottom) shows that the magnetic beads are gradually focused onto the sidewalls of the channel by a combination of positive magnetophoresis and hydrophoresis. (c) Optical micrographs showing the particle trajectories at the outlet at a flow rate of $1,5,10$ and $20 \mu \mathrm{min}^{-1}$. (d) The focusing efficiency of the magnetic beads measured in the "regular" setup. The average value was 3 times the measurement and the error bar represents standard deviation. (e) The plot of the calculated gradients of the square of the magnetic field in different setups. 
setup, the focusing efficiency is similar to the "flipped" setup and on which the flow rate has an adverse effect. The focusing efficiency in this "regular" setup dropped from $73.5 \pm 4.1 \%$ to $46.6 \pm 6.8 \%$ as the flow rate increased from 1 to $20 \mu \mathrm{l} \mathrm{min}{ }^{-1}$. This shorter retention time for the beads passing through the channel is one reason for this phenomenon because at a higher flow rate, the magnetic beads do not have enough time to migrate towards the grooves under a magnetic field, and thus their focusing performance is poor.

The biggest difference between these two setups lies in the magnitude of gradient of the magnetic fields. The distance between the channel and the magnets was $1 \mathrm{~mm}$ (i.e., the thickness of the glass side) in the "flipped" setup, whereas this value increased to $\sim 3 \mathrm{~mm}$ (the thickness of PDMS) when the magnets were placed on top of the PDMS in the "regular" setup (Fig. 3(b)). To study this magnetic field that decreases as the distance increases, a 2D model was built to simulate how the magnetic field is distributed (see Fig. S1 in the supplementary material). Based on this model, the gradients of the square of the magnetic field along the height of the channel at different setups were calculated numerically (Fig. 3(e)). The average intensity of the magnetic field gradient in the "flipped" setup was $\sim 2$ times as high as in the "regular" setup, and therefore the beads experiencing a weaker magnetic force in the "regular" setup were prone to have less focusing efficiency.

The good focusing performance in the "flipped" setup meant that this setup was used for the magnetophoretic separation of magnetic and non-magnetic particles. To create the differences in magnetic susceptibility, $6 \mu \mathrm{m}$ magnetic beads and $13 \mu \mathrm{m}$ polystyrene particles were suspended in the $\times 0.05$ ferrofluid. This particle-laden sample was then injected into the micro-channel with a flow rate that varied from 5 to $80 \mu \mathrm{min}^{-1}$, to examine how this variable flow rate affected the particle separation. The transverse positions for each type of particle at the outlet were measured statistically as a probability distribution function (PDF). ${ }^{24}$ The PDF is quantified by particle counting.

Fig. 4(a) shows that the magnetic beads focused to a stream near the centreline of the channel by balancing the magnetic force and drag force, while the non-magnetic beads moved along the sidewalls of the channel in a combination

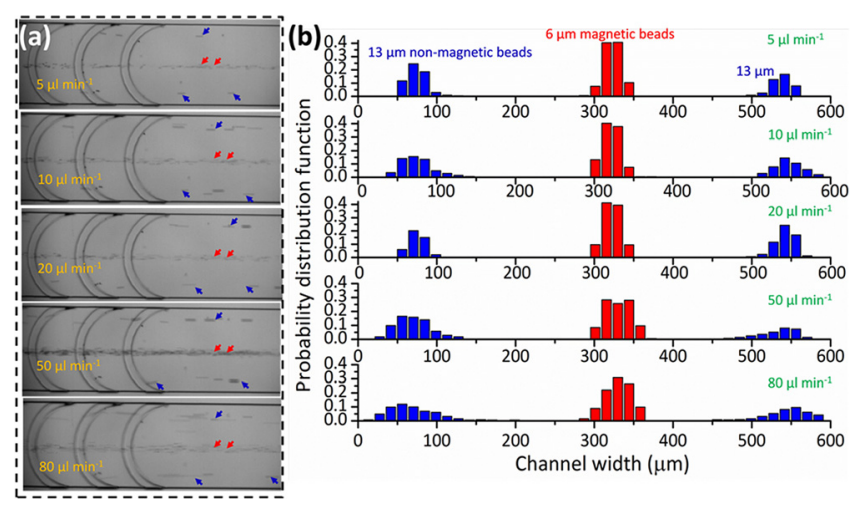

FIG. 4. (a) The effect that flow rate has on the separation of magnetic and non-magnetic beads in the "flipped" setup. The red arrows point to the $6 \mu \mathrm{m}$ magnetic beads, while the blue ones highlight the $13 \mu \mathrm{m}$ non-magnetic beads. (b) A plot of particle PDF at the channel outlet. More than 500 events were counted for each type of particle. of hydrophoresis and negative magnetophoresis. Because the equilibrium positions in the groove-based channel are distinct, those particles with different magnetic properties become widely separated. The PDF of magnetic beads in a diluted ferrofluid do not change very often within the flow rate of $50 \mu \mathrm{l} \mathrm{min}^{-1}$ (Fig. 4(b)), a result that is identical to the results reported above. Since the flow rate caused the hydrophoretic focusing to deteriorate, as reported in our previous work, ${ }^{22}$ the non-magnetic beads tended towards having a wider distribution at the outlet at a higher flow rate (Fig. 4(b)), whereas the magnetic beads were clearly separated from the non-magnetic beads because the magnetic and nonmagnetic beads did not overlap with each other, even at a higher flow rate. To justify the separation, the trajectories of $13 \mu \mathrm{m}$ beads were captured under the fluorescent field (see Fig. S2 in the supplementary material), which demonstrated that $6 \mu \mathrm{m}$ magnetic beads and $13 \mu \mathrm{m}$ polystyrene particles had no overlap.

Although the flow rates in this work are less than $100 \mu \mathrm{l}$ $\min ^{-1}$, throughput can be improved by increasing the width of the channel because the focusing mechanism for the magnetic and non-magnetic beads is related to the height of the channel. Alternatively, integrating $N$ columns of grooves into a single chip can create $2 N+1$ parallel focused streams of particles (see Fig. S3 in the supplementary material); this is an easy and very efficient way to improve the throughput of the channel.

In summary, we have now demonstrated a highthroughput, sheathless, magnetophoretic separation of magnetic and non-magnetic beads in a groove-based channel. It has also been found that magnetic beads have different particle trajectories in different setups. For example, in the "flipped" setup, the magnetic beads were focused onto the centreline of the channel and retained a high focusing efficiency $(\sim 85 \%)$ at a flow rate of $\sim 100 \mu \mathrm{l} \mathrm{min}^{-1}$; in fact the focusing efficiency is insensitive to the flow rate so this device can be operated manually; whereas the magnetic beads in the "regular" setup formed hydrophoretic ordering due to positive magnetophoresis and focused onto the sidewalls of the channel. Finally, the magnetic and non-magnetic beads were suspended in a diluted ferrofluid to generate the mismatch of magnetic susceptibility. This separation was achieved in the "flipped" setup where the magnetic beads stayed near the centreline of the channel by balancing the magnetic force and drag force, and the non-magnetic beads moved along the sidewalls of the channel due to hydrophoresis and negative magnetophoresis. The particle distribution at the outlet revealed a clear separation between the magnetic and non-magnetic particles, even at a flow rate of $80 \mu \mathrm{l}$ $\min ^{-1}$. Furthermore, the throughput of the groove-based channel can be enhanced considerably by integrating multiple columns of grooves into a single chip for parallel processing.

See supplementary material for the details of materials and figures of simulated magnetic field and $13 \mu \mathrm{m}$ particle trajectories under fluorescent field.

This work is supported by the University of Wollongong-China Scholarship Council joint scholarships. 
${ }^{1}$ S. Kim, S.-I. Han, M.-J. Park, C.-W. Jeon, Y.-D. Joo, I.-H. Choi, and K.-H. Han, Anal. Chem. 85, 2779-2786 (2013).

${ }^{2}$ Y.-H. Lin, C.-C. Wang, and K. F. Lei, Biomed. Microdevices 16, 199-207 (2014).

${ }^{3}$ A. H. C. Ng, U. Uddayasankar, and A. R. Wheeler, Anal. Bioanal. Chem. 397, 991-1007 (2010).

${ }^{4}$ J. H. Shin, M. G. Lee, S. Choi, and J.-K. Park, RSC Adv. 4, 39140-39144 (2014).

${ }^{5}$ M. S. Kim, T. S. Sim, Y. J. Kim, S. S. Kim, H. Jeong, J.-M. Park, H.-S. Moon, S. I. Kim, O. Gurel, and S. S. Lee, Lab Chip 12, 2874-2880 (2012).

${ }^{6}$ C. W. Shields IV, L. M. Johnson, L. Gao, and G. P. López, Langmuir 30, 3923-3927 (2014).

${ }^{7}$ T. Yasukawa, M. Suzuki, T. Sekiya, H. Shiku, and T. Matsue, Biosens. Bioelectron. 22, 2730-2736 (2007)

${ }^{8}$ M. Hejazian, W. Li, and N.-T. Nguyen, Lab Chip 15, 959-970 (2015).

${ }^{9}$ T. Zhu, R. Cheng, Y. Liu, J. He, and L. Mao, Microfluid. Nanofluid. 17, 973-982 (2014).

${ }^{10}$ N. Xia, T. P. Hunt, B. T. Mayers, E. Alsberg, G. M. Whitesides, R. M. Westervelt, and D. E. Ingber, Biomed. Microdevices 8, 299-308 (2006).
${ }^{11}$ F. Del Giudice, H. Madadi, M. M. Villone, G. D'Avino, A. M. Cusano, R. Vecchione, M. Ventre, P. L. Maffettone, and P. A. Netti, Lab Chip 15, 1912-1922 (2015).

${ }^{12}$ S. Choi, S. Song, C. Choi, and J. K. Park, Small 4, 634-641 (2008).

${ }^{13}$ P. Sajeesh and A. K. Sen, Microfluid. Nanofluid. 17, 1-52 (2014).

${ }^{14}$ A. D. Stroock, Science 295, 647-651 (2002).

${ }^{15}$ N. Gadish and J. Voldman, Anal. Chem. 78, 7870-7876 (2006).

${ }^{16}$ C. H. Hsu, D. Di Carlo, C. Chen, D. Irimia, and M. Toner, Lab Chip 8, 2128-2134 (2008).

${ }^{17}$ B. Massey, Mechanics of fluids (Chapman \& Hall, London, 1989).

${ }^{18}$ S. A. Peyman, E. Y. Kwan, O. Margarson, A. Iles, and N. Pamme, J. Chromatogr., A 1216, 9055-9062 (2009).

${ }^{19}$ S. Yan, J. Zhang, H. Chen, D. Yuan, G. Alici, H. Du, Y. Zhu, and W. Li, Biomed. Microdevices 18, 54 (2016).

${ }^{20}$ S. Choi and J. K. Park, Anal. Chem. 80, 3035-3039 (2008).

${ }^{21}$ S. Choi and J.-K. Park, Lab Chip 7, 890-897 (2007).

${ }^{22}$ S. Yan, J. Zhang, M. Li, G. Alici, H. Du, R. Sluyter, and W. Li, Sci. Rep. 4, 5060 (2014).

${ }^{23}$ S. Yan, J. Zhang, G. Alici, H. Du, Y. Zhu, and W. Li, Lab Chip 14, 2993-3003 (2014).

${ }^{24}$ X. Lu, L. Zhu, R.-M. Hua, and X. Xuan, Appl. Phys. Lett. 107, 264102 (2015). 